\title{
Upper-Extremity Deep Venous Thrombosis: Analysis of 348 Cases
}

\author{
๑ İbrahim Erdinç, ๑ Mustafa Barış Kemahlı
}

İzmir Bozyaka Eğitim ve Araştırma Hastanesi, Clinic of Cardiovascular Surgery, İzmir, Turkey

\begin{abstract}
Objectives: In this study, we aimed to determine the clinical features and outcomes of patients with upper-extremity deep venous thrombosis (UEDVT).

Materials and Methods: Three hundered and fourty eight cases diagnosed with UEDVT were included in the study. Risk factors, symptoms, thrombosis localization, and clinical outcomes were examined.

Results: The most common risk factors were central venous catheter (CVC) $(82 \%)$ and malignancy (45\%). Edema (77\%) and pain (56\%) were the reasons for the admission of patients. The most common site involved was the subclavian vein. Although the frequency of pulmonary embolism was $4 \%$, the rate of 60 -day mortality was determined as $9 \%$.

Conclusion: CVC and the presence of malignancy are common risk factors for UEDVT. Despite its low frequency of pulmonary embolism, it has non-low mortality associated with possible underlying diseases.

Keywords: Deep vein thrombosis, upper extremity, low molecular weight heparin, central venous catheter, medical treatment
\end{abstract}

\section{Introduction}

Upper-extremity deep venous thrombosis (UEDVT) is called internal jugular, brachiocephalic, brachial, subclavian, axillary veins thrombosis ${ }^{(1)}$. It is divided into two etiological classifications, as primary and secondary. Primary UEDVT is defined as spontaneous thrombosis of the subclavian and axillary vein. It includes PagetSchroetter syndrome, which is defined as venous thoracic outlet syndrome ${ }^{(2)}$. Thoracic outlet abnormalities may

Address for Correspondence: İbrahim Erdinç, İzmir Bozyaka Eğitim ve Araştırma Hastanesi, Clinic of Cardiovascular Surgery, İzmir, Turkey E-mail: patentheart@yahoo.com ORCID: orcid.org/0000-0003-4354-5396

Received: 26.06.2021 Accepted: 19.08.2021

Cite this article as: Erdinç İ, Kemahlı MB. Upper-Extremity Deep Venous Thrombosis: Analysis of 348 Cases.

EJCM 2021;9(3):130-135.

DOI: 10.32596/ejcm.galenos.2021-06-039 
occur without an identifiable cause or may be caused by upper extremity movements that create vascular stress causing intimal damage ${ }^{(3)}$. There is an underlying facilitating reason in secondary UEDVTs. These are central venous catheter (CVC) insertion, malignancy, coagulation abnormalities, and genetic risk factors ${ }^{(4)}$. In this study, we aimed to determine the risk factors, clinical characteristics, and outcomes of patients diagnosed with UEDVT in our clinic

\section{Materials and Methods}

This study was approved by Bozyaka Training and Research Hospital Ethics Review Board in accordance with the Declaration of Helsinki (date: 28.05.2022, protocol no: 2021/92). In our study, we retrospectively examined patients older than 18 years of age, who were diagnosed with UEDVT by Doppler USG, contrast venography, or CTA between November 2001 and August 2019. We recorded the patients' basic clinical characteristics, comorbidity, and risk factors for UEDVT. Symptoms and localization of deep vein thrombosis were determined. Clinical outcomes were determined in terms of pulmonary embolism, mortality, post-thrombotic syndrome (PTS), and recurrence of UEDVT. Considering cancer and other clinical characteristics of the patients, Low-molecular-weight heparin (LMWH) and/or warfarin was administered for three months as a treatment protocol.

\section{Results}

During this period, 348 cases were detected. Table 1 shows the clinical characteristics and treatment parameters of these cases. Forty-seven percent of these cases were women with an average age of 57 years. CVC was present in $82 \%$ of the cases. The second most common UEDVT risk factor was the presence of malignancy. Twelve percent of the patients had a history of lower-extremity deep venous thrombosis (LEDVT) in the last year. The proportion of patients receiving anticoagulant therapy was 36\%.

Symptoms and their distribution are given in Table 2. The most common reason for admission was swelling in the arm and neck (77\%). Fifty-six percent of the patients complained of pain at the time of presentation. A lesser of them applied with the complaints of discoloration such as bruising and redness in the extremity. The average admission time to the hospital was 2-6 days and 3.5 days on average.

Table 3 shows the anatomical involvement of UEDVT cases. The most commonly involved venous structure was the subclavian vein. Its continuation, the axillar vein, was the second most frequently affected site. The internal

Table 1. Clinical characteristics and treatment parameters of the cases

\begin{tabular}{|l|l|}
\hline Features & $\mathbf{n}$ \\
\hline Age & 57 \\
\hline Gender (female) & 47 \\
\hline Central venous catheter & 82 \\
\hline Malignancy & 45 \\
\hline Hemodialysis catheter & 14 \\
\hline Surgery and trauma & 10 \\
\hline $\begin{array}{l}\text { Immobility } \\
\text { LEDVT history (in the last year) }\end{array}$ & 8 \\
\hline Anticoagulant therapy & 12 \\
\hline $\begin{array}{l}\text { Those who do not receive anticoagulant } \\
\text { treatment }\end{array}$ & 64 \\
\hline LEDVT: Lower-extremity deep venous thrombosis, & $n$ : Number \\
\hline
\end{tabular}

Table 2. Symptoms and their distribution

\begin{tabular}{|l|l|}
\hline Symptoms & $\mathbf{n}$ \\
\hline Edema & 77 \\
\hline Pain & 56 \\
\hline Color change & 19 \\
\hline n: Number & \\
\hline
\end{tabular}

Table 3. Anatomical involvement of UEDVT cases

\begin{tabular}{l|l}
\hline Involvement & $\%$ \\
\hline Superior vena cava & 1 \\
\hline Innominate vein & 1 \\
\hline Internal juguler vein & 40 \\
\hline Subclavian vein & 66 \\
\hline Aksiller vein & 51 \\
\hline Brakial vein & 19 \\
\hline UEDVT: Upper-extremity deep venous thrombosis, $n$ : Number
\end{tabular}


jugular vein, which is an important central venous access site, was the third most common UEDVT location with the rate of $40 \%$. Intrathoracic central venous structures including the innominate vein and SVC were the least affected structures with the rate of $1 \%$.

Clinical outcomes are shown in Table 4. Pulmonary embolism was at a low frequency of $4 \%$. We determined the sixty-day mortality as $9 \%$. The rate of PTS with UEDVT morbidity was about $8 \%$ ( $8 \%$ of patients with the PTS that causes ongoing swelling, pain, loss of vascular access in the extremity with UEDVT morbidity). In our study, UEDVT had a recurrence rate of $12 \%$.

\section{Discussion}

Although UEDVT is uncommon, its frequency is increasing with advanced treatment methods and increased invasive procedures ${ }^{(2,5)}$. In a study involving 11,564 deep vein thrombosis patients, the incidence of UEDVT was found to be $4.4 \%{ }^{(6)}$. It has historically been considered a benign disease. However, with the demonstration that it causes serious complications such as pulmonary embolism, superior vena cava syndrome, loss of vascular access, post-thrombotic venous insufficiency, its importance has increased Upper extremity deep vein thrombosis is extremely rare compared to lower extremity deep venous thrombosis ${ }^{(7)}$. The main reasons for this can be counted as the arm veins are less exposed to the effect of gravity and have fewer valves. To reveal the etiological and clinical features of UEDVT disease, which causes morbidity and morbidity, will help to understand the disease and its causes. The most common complaints in patients presenting with a diagnosis of UEDVT are pain

Table 4. Clinical outcomes

\begin{tabular}{|l|l|}
\hline Clinical outcomes & $\mathbf{n}$ \\
\hline Pulmonary embolism & 4 \\
\hline Mortality (60 days) & 9 \\
\hline Post thrombotic syndrome & 8 \\
\hline UEDVT recurrence & 12 \\
\hline UEDVT: Upper-extremity deep venous thrombosis, $n$ : Number
\end{tabular}

and swelling in the $\operatorname{arm}^{(8)}$. In addition, other application complaints are erythema, neck and face swelling. A significant $33-60 \%$ of patients with secondary UEDVT do not express symptoms. In our study, patients diagnosed with UEDVT most frequently presented with swelling and arm pain.

In our study, as in the studies in the literature, the most prominent risk factor was permanent catheter placement. Eighty-two percent of the patients with UEDVT had intravenous instrumentation such as $\mathrm{CVC}$, pacemakers/ defibrillators. With increased CVC placement and widespread use of VDU as a diagnostic tool, UEDVT patients are more $\operatorname{common}^{(9)}$. Catheter-induced UEDVT has been associated with catheter size. It has been reported that as the catheter size increases, the frequency of UEDVT increases $^{(10)}$. In addition, Gonsalves et al. ${ }^{(10)}$ showed that the longer the catheter residence time, the higher the frequency of central vein stenosis and occlusion ${ }^{(11,12)}$. Thrombosis was detected in $10 \%$ of the patients with pacemakers. This thrombosis frequency increases as the number of pacemakers lead increases ${ }^{(13)}$. Sixty-nine percent of UEDVTs occurring in cancer patients are CVC-related thrombosis ${ }^{(14)}$. De Cicco et al. ${ }^{(15)}$ identified the first days and left subclavian artery catheterization as risk factors for UEDVT in malignant patients with CVC.

The second important risk factor shown in our study was the presence of cancer ${ }^{(16)}$. The rate of patients accompanied by cancer was $45 \%$. Although cancer itself creates a prothrombotic process, chemotherapy, hormone replacement therapy, and medical status of cancer patients lay the groundwork for UEDVT ${ }^{(17,18)}$.

In a prospective study involving cancer patients with CVC, symptomatic UEDVT was found at the rate of $6 \%{ }^{(15)}$. In a study conducted with a population that does not have CVC but is known to have cancer.UEDVT in patients with active cancer, 18 times than in those without cancer, those with a history ofcancer were found to have a 7.7-fold increased risk $^{(19)}$. In another study involving cancer patients, it was reported that UEDVT cases had a high recurrence rate of $18 \%{ }^{(20)}$. Malignancy and catheterrelated thrombosis are associated with recurrence ${ }^{(21)}$. 
PTS is one of the morbidities of UEDVT and although its frequency is between 6 and 37\%, Thiyagarajah et al. ${ }^{(22)}$ associated it more with primary UEDVT, reporting it as $14 \%$ for secondary UEDVT. Venous hypertension due to venous thrombus that persists in PTS occurs as a result of damage to the venous valves (especially for the lower extremity) and the deterioration of the microcirculation due to congestion and edema. In our study, the frequency of PTS was found to be $8 \%$.

Recurrence and major bleeding rates in UEDVT patients are similar to those in LEDVT patients ${ }^{(23,24)}$. This reveals the need and necessity for treatment ${ }^{(25)}$. However, similar major bleeding frequency requires treatment follow-up as in LEDVT patients.

In a recent study evaluating the treatment approaches of clinicians in UEDVT cases, it was observed that the management of UEDVT patients differed greatly among physicians, only $10 \%$ of the physicians adopted the current guidelines and less of them applied the treatment by them. Possibly due to the low quality of data on UEDVT thrombosis, no recommendations were made in the latest American College of Chest Physicians (ACCP) guideline. Considering all these, the lack of research and data on UEDVT prevents the formation and applicability of a treatment consensus.

Its treatment was mainly based on data obtained from $\operatorname{LEDVT}^{(25)}$. The main purpose of the treatment is to alleviate the symptoms of acute UEDVT, to prevent PE and late complications. Medical treatment was applied to our patients, interventional mechanical treatments and surgical treatment were not applied. In our study, we determined the treatment principle of anticoagulation with LMWH or warfarin for three months and/or removal of the catheter in the treatment of our patients. By the ACCP recommendations, patients with cancer were anticoagulated with LMWH and, if not, with warfarin. The rate of patients we followed up with anticoagulation was $64 \%$. During the follow-up, no bleeding problem related to GIS was encountered in our patients, h2 resp antagonist treatment was routinely added to the treatment of the patients and the patients were informed about the hematemesis or melena that may be associated with bleeding. In patients with catheter-associated UEDVT, catheter removal was decided by considering the individual characteristics of the patient, such as the presence of infection, the need for vascular access, the functionality of the catheter, anticoagulant therapy contraindication, response to treatment, and the severity of symptoms. There are different rates of pulmonary embolism in the literature. Although a high incidence of $36 \%$ was reported in previous studies, Levy et al. ${ }^{(26)}$ found a low incidence of PE (2\%) independent of anticoagulant therapy. In another similar study, in which they used DMAH and warfarin as anticoagulant treatment, although only $40 \%$ of 300 patients were anticoagulated, they did not recommend routine anticoagulation to prevent PE due to hemorrhagic complications ${ }^{(27)}$. Beiswenger et al. ${ }^{(28)}$, found that mortality was higher in the group that did not receive anticoagulant treatment. However, they associated the data with comorbid and demographic characteristics that prevent patients from receiving anticoagulant therapy rather than pulmonary embolism. They found the 30-day and 6-month mortality rates to be $16.55 \%$ and $27.5 \%$, respectively ${ }^{(28)}$. Age, dialysis, central location, ischemic stroke after diagnosis, and cancer at the time of diagnosis have been shown as risk factors for clinical outcomes ${ }^{(28)}$. In our study, the rate of pulmonary embolism was found to be $4 \%$, and the two-month mortality was $9 \%$. The prognosis of the disease is closely related to the prognosis of cancer, as in our study of the underlying chronic disease.

In the COVID-19 pandemic, we are in, there are case reports diagnosed with UEDVT. Considering the hypercoagulable state caused by SARS-CoV-2 infection, it will not be surprising that it is included in the etiology of UEDVT ${ }^{(29)}$.

\section{Conclusion}

UEDVT is a less common disease with a thrombotic process than LEDVT. CVC and malignancy are the most common risk factors for UEDVT. UEDVT should be kept 
in mind especially in patients suffering from pain and swelling with the stated risk factors.

Despite its low prevalence of PE, it probably has nonlow mortality associated with underlying diseases such as malignancy. Close follow-up should be carried out in terms of UEDVT in patients who received or were diagnosed with a treatment with $\mathrm{CVC}$, and patients should be told that such a complication may occur. In addition, as soon as the symptoms of the disease begin, they should be told to apply to the nearest hospital at an early stage. Anticoagulant treatment should be applied due to the risk of PTS and recurrence.

\section{Acknowledgments}

We sincerely thank Murat Uğurlucan, Medical Faculty Department of Cardiovascular Surgery, İstanbul Medipol University and Fulya Y1lmaz, University of Health Sciences Turkey, İzmir Bozyaka Training and Research Hospital, Clinic of Anesthesiology and Reanimation, İzmir, for their comments on the manuscript.

\section{Ethics}

Ethics Committee Approval: This study was approved by Bozyaka Training and Research Hospital Ethics Review Board in accordance with the Declaration of Helsinki (date: 28.05.2022, protocol no: 2021/92).

Informed Consent: This study does not require patient consent.

Peer-review: Internally and externally peer-reviewed.

\section{Authorship Contributions}

Surgical and Medical Practices: İ.E., M.B.K., Concept: İ.E, M.B.K., Design: İ.E., M.B.K., Data Collection or Processing: İ.E., M.B.K., Analysis or Interpretation: İ.E., M.B.K., Literature Search: İ.E., M.B.K., Writing: İ.E., M.B.K.

Conflict of Interest: The authors declare that they have no conflict of interest.
Financial Disclosure: The authors declare no financial support by any grant or research sponsor and no competing financial interest.

\section{References}

1. Bleker SM, van Es N, Kleinjan A, et al. Current management strategies and long-term clinical outcomes of upper extremity venous thrombosis. J Thromb Haemost 2016;14:973-81.

2. Sajid MS, Ahmed N, Desai M, Baker D, Hamilton G. Upper limb deep vein thrombosis: a literature review to streamline the protocol for management. Acta Haematol 2007;118:10-8.

3. Czihal M, Hoffmann U. Upper extremity deep venous thrombosis. Vasc Med 2011;16:191-202.

4. Kearon C, Kahn SR, Agnelli G, Goldhaber S, Raskob GE, Comerota AJ Antithrombotic therapy for venous thromboembolic disease: American College of Chest Physicians Evidence-Based Clinical Practice Guidelines (8th Edition). Chest 2008;133(6 Suppl):454S-545S.

5. Joffe HV, Kucher N, Tapson VF, Goldhaber SZ; Deep Vein Thrombosis (DVT) FREE Steering Committee. Upper-extremity deep vein thrombosis: a prospective registry of 592 patients. Circulation 2004;110:1605-11.

6. Muñoz FJ, Mismetti P, Poggio R, et al. Clinical outcome of patients with upper-extremity deep vein thrombosis: results from the RIETE Registry. Chest 2008;133:143-8.

7. Becker DM, Philbrick JT, Walker FB 4th. Axillary and subclavian venous thrombosis. Prognosis and treatment. Arch Intern Med 1991;151:1934-43.

8. Marnejon T, Angelo D, Abu Abdou A, Gemmel D. Risk factors for upper extremity venous thrombosis associated with peripherally inserted central venous catheters. J Vasc Access 2012;13:231-8.

9. Evans RS, Sharp JH, Linford LH, et al. Risk of symptomatic DVT associated with peripherally inserted central catheters. Chest 2010;138:803-10.

10. Gonsalves CF, Eschelman DJ, Sullivan KL, DuBois N, Bonn J. Incidence of central vein stenosis and occlusion following upper extremity PICC and port placement. Cardiovasc Intervent Radiol 2003;26:123-7.

11. Korkeila P, Nyman K, Ylitalo A, et al. Venous obstruction after pacemaker implantation. PACE - Pacing and Clinical Electrophysiology. Pacing Clin Electrophysiol 2007;30:199-206.

12. Rozmus G, Daubert JP, Huang DT, Rosero S, Hall B, Francis C. Venous thrombosis and stenosis after implantation of pacemakers and defibrillators. J Interv Card Electrophysiol 2005;13:9-19.

13. de Cicco M. The prothrombotic state in cancer: Pathogenic mechanisms. Critical Reviews in Oncology/Hematology. Crit Rev Oncol Hematol 2004;50:187-96.

14. ALKindi SY, Chai-Adisaksopha C, Cheah M, Linkins LA. Management of cancer-associated upper extremity deep vein thrombosis with and without venous catheters at a tertiary care center. Thromb Res 2018;166:92-95.

15. de Cicco M, Matovic M, Balestreri L, et al. Central venous thrombosis: An early and frequent complication in cancer patients bearing long-term 
silastic catheter. A prospective study. Thrombosis Research. 1997;86:10113 .

16. Prandoni P, Lensing AWA, Piccioli A, et al. Recurrent venous thromboembolism and bleeding complications during anticoagulant treatment in patients with cancer and venous thrombosis. Blood 2002;100:3484-8

17. Blom JW, Doggen CJ, Osanto S, Rosendaal FR. Old and new risk factors for upper extremity deep venous thrombosis. J Thromb Haemost 2005;3:24718 .

18. Gouin-Thibault I, Achkar A, Samama MM. The Thrombophilic State in Cancer Patients. Acta Haematol 2001;106:33-42.

19. Shankar L, Junaid H.A Rare Finding of Upper Limb Deep Venous Thrombosis in a Patient with COVID-19. J Coll Physicians Sur Pak 2020;30:76-7.

20. ALKindi SY, Chai-Adisaksopha C, Cheah M, Linkins LA. Management of cancer-associated upper extremity deep vein thrombosis with and without venous catheters at a tertiary care center. Thromb Res 2018;166:92-5.

21. Cote LP, Greenberg S, Caprini JA, et al. Comparisons Between Upper and Lower Extremity Deep Vein Thrombosis: A Review of the RIETE Registry. Clin Appl Thromb Hemost 2017;23:748-54.

22. Thiyagarajah K, Ellingwood L, Endres K, et al. Post-thrombotic syndrome and recurrent thromboembolism in patients with upper extremity deep vein thrombosis: A systematic review and meta-analysis. Thromb Res 2019;174:34-39.
23. Spencer FA, Emery C, Lessard D, Goldberg RJ; Worcester Venous Thromboembolism Study. Upper extremity deep vein thrombosis: a community-based perspective. Am J Med 2007;120:678-84.

24. Muñoz FJ, Mismetti P, Poggio R, et al. Clinical outcome of patients with upper-extremity deep vein thrombosis: results from the RIETE Registry. Chest 2008;133:143-8.

25. Schmittling ZC, McLafferty RB, Bohannon WT, Ramsey DE, Hodgson KJ. Characterization and Probability of Upper Extremity Deep Venous Thrombosis. Annals of Vascular Surgery. Springer New York 2004;18:5527.

26. Levy MM, Albuquerque F, Pfeifer JD. Low incidence of pulmonary embolism associated with upper-extremity deep venous thrombosis. Ann Vasc Surg 2012;26:964-72.

27. Heil J, Miesbach W, Vogl T, Bechstein WO, Reinisch A. Deep Vein Thrombosis of the Upper Extremity. Dtsch Arztebl Int 2017;114:244-9.

28. Beiswenger AC, Quereshy HA, Rouabhi M, et al. Midterm outcomes in patients with upper extremity deep vein thrombosis. J Vasc Surg Venous Lymphat Disord 2020;8:930-8.e2.

29. Di Nisio M, Van Sluis GL, Bossuyt PM, Büller HR, Porreca E, Rutjes AW. Accuracy of diagnostic tests for clinically suspected upper extremity deep vein thrombosis: a systematic review. J Thromb Haemost 2010;8:684-92. 